\title{
Transatlantica
}

Revue d'études américaines. American Studies Journal

\section{L'énigme du couple « race et classe » dans la société et les sciences sociales étasuniennes *}

\section{Loïc Wacquant}

\section{OpenEdition}

1 Journals

\section{Édition électronique}

URL : https://journals.openedition.org/transatlantica/4359

DOI : $10.4000 /$ transatlantica.4359

ISSN : 1765-2766

Éditeur

Association française d'Etudes Américaines (AFEA)

\section{Référence électronique}

Loïc Wacquant, «L'énigme du couple « race et classe » dans la société et les sciences sociales étasuniennes * », Transatlantica [En ligne], 1 | 2009, mis en ligne le 03 juillet 2009, consulté le 15 septembre 2021. URL : http://journals.openedition.org/transatlantica/4359 ; DOI : https://doi.org/ 10.4000/transatlantica.4359

Ce document a été généré automatiquement le 15 septembre 2021.

\section{c) (†) $९$}

Transatlantica - Revue d'études américaines est mise à disposition selon les termes de la licence Creative Commons Attribution - Pas d'Utilisation Commerciale - Pas de Modification 4.0 International. 


\title{
L'énigme du couple « race et classe » dans la société et les sciences sociales étasuniennes *
}

\author{
Loïc Wacquant
}

\section{NOTE DE L'AUTEUR}

* Cet article est la traduction de « The Puzzle of Race and Class in American Society and Social Science", Benjamin E. Mays Monographs, vol. 2, no. 1 (automne 1989), pp. 7-20, texte basé sur une communication au Symposium Benjamin E. Mays sur "Race: Declining or Increasing Significance?", Indiana University of Pennsylvania, 14 et 15 octobre 1988. Il est traduit de l'américain par Isabelle Richet.

Avec les théories raciales, on peut prouver ou réfuter tout ce que l'on veut.

Max Weber. ${ }^{1}$

1 L'appartenance raciale demeure-t-elle un facteur conséquent aux États-unis vingt-cinq ans après la révolution des droits civiques, et si oui dans quelle mesure ? Ou bien la classe l'a-t-elle remplacée, devenant la seule force qui détermine désormais la trajectoire sociale des Noirs américains? Telles sont les questions que nous proposons de traiter ici. Comme l'a montré Reynolds Farney dans son ouvrage Blacks and Whites: Narrowing the Gap ?, il y a trois façons de répondre à ces interrogations et de nombreux travaux de recherche dont les données convaincantes peuvent être mobilisées pour étayer chacune d'entre elles, car au cours du dernier quart de siècle l'évolution des inégalités raciales a été contradictoire et fluctuante. ${ }^{2}$

2 Les optimistes affirment que la couleur de peau ne contrevient plus à l'égalité des chances dans la société étatsunienne, et pour appuyer cette affirmation ils peuvent, de façon tout à fait légitime, signaler le déclin substantiel des différences raciales dans les niveaux d'éducation, l'amélioration spectaculaire des positions occupées par les Noirs 
ayant un emploi, la hausse notable du revenu annuel des travailleurs issus des minorités, et même la baisse des hostilités raciales ouvertes et la perte de légitimité des attitudes discriminatoires. Les pessimistes, de leur côté, rejettent ces tendances et soulignent que dans aucun de ces domaines les Noirs n'ont atteint la parité avec les Blancs ; ils soulignent également que les taux de chômage, de participation à la force de travail, de pauvreté des familles et des enfants ont en fait régressé et non progressé pour les Noirs, tandis que la ségrégation résidentielle et scolaire dans les grandes métropoles n'a pratiquement pas changé depuis les années soixante. Une troisième façon de voir les choses cherche à réinterpréter ces mêmes données comme autant de preuves que l'Amérique noire est en train de devenir de plus en plus polarisée entre, d'un côté, une classe moyenne relativement stable de Noirs éduqués à l'université qui ont eu accès à l'ensemble de biens et de services qui incarnent le "rêve américain », et qui sont de plus en plus à même de transmettre leur statut privilégié à leurs enfants ; de l'autre un sous-prolétariat opprimé et appauvri, piégé dans des quartiers déshérités totalement ravagés et n'ayant pas d'autre perspective que l'accroissement de la marginalisation, du désespoir et de la violence quotidienne qui affligent les ghettos contemporains. $^{3}$

3 Ce qui soulève la question: si ces trois lectures - l'optimiste, la pessimiste et la thèse de la polarisation- peuvent être jugées correctes au même moment, c'est peut-être parce que la question posée n'est pas la bonne, ou qu'elle n'est pas formulée d'une façon scientifiquement pertinente. Dans La Formation de l'esprit scientifique, l'épistémologue et historien des sciences Gaston Bachelard montre que seuls des problèmes scientifiques sont susceptibles de recevoir des réponses scientifiques. La thèse centrale de cet article est que, nonobstant sa brève et illustre carrière, la controverse autour de l'importance décroissante de la race est un faux problème qui ne peut, sous cette forme, être résolu scientifiquement et doit donc avant tout être reformulé.

Cette reformulation doit éviter trois erreurs récurrentes qui ont contaminé l'étude de la race dans les sciences sociales américaines: l'essentialisme (la conviction que la discrimination raciale est dotée d'un caractère immuable et qu'elle affecte nécessairement la totalité du système social); le déterminisme objectiviste (l'idée que race ou classe sont des facteurs purement « objectifs » qui déterminent les positions et les conduites indépendamment des expériences subjectives et des représentations des agents); enfin le réductionnisme unidimensionnel (l'idée implicite qu'un principe unique contrôle nécessairement la production des faits sociaux). A l'inverse, elle doit mettre au centre de la problématique trois ingrédients fructueux dans l'histoire des sciences sociales qui font cruellement défaut dans ce débat : la réflexivité, la complexité historique, et la notion d'agence. Bachelard a aussi écrit que "le simple est toujours le simplifié " et si je ne peux faire passer qu'un seul message dans ce court texte, c'est que la problématique de la race et de sa relation à la classe (et à d'autres principes de domination, notamment le genre) est beaucoup plus compliquée que le débat ne le laisse entendre. Et cette complexité découle en grande partie du chevauchement qui existe, dans ce cas, entre sujet et objet, ainsi que du fait que l'action historique a joué un rôle considérable dans la création et la reproduction des inégalités raciales -bien plus important que ne l'admettent les théories existantes. L'exergue de Max Weber est un appel à la nécessité de complexifier notre compréhension du rapport entre race et classe en problématisant chacun des deux termes et leur interaction. 


\section{La thèse de Wilson, ou " l'importance croissante de la classe »}

5 Ces réflexions s'articulent autour de l'étude désormais classique de Wilson, The Declining Significance of Race, qui provoqua une controverse vigoureuse et parfois malveillante, lors de sa publication en 1978 par les presses de l'Université de Chicago. ${ }^{4}$ Dans la mesure où ce livre a été tellement déformé ou fort mal lu, je vais d'abord en récapituler les principales affirmations. La thèse centrale de Wilson, telle que je l'interprète, est essentiellement historique, couvrant un siècle de rapports entre Noirs et Blancs en Amérique. Cette thèse affirme qu'un ensemble de changements interdépendants dans la structure économique, l'autonomie de l'État et la politique publique aux États-unis ont fait passer les relations raciales à travers trois phases distinctes et ont progressivement fait de la position de classe un déterminant de plus en plus important des chances de vie des individus noirs. ${ }^{5}$

6 Dans la première phase, celle de l'oppression raciale de caste associée à l'économie de plantation dans le Sud avant la guerre civile, le système de production était dominé par une élite hégémonique de planteurs blancs qui laissait effectivement les autres Blancs sans pouvoir et réduisait les Noirs à l'esclavage, tandis que l'État servait d'instrument d'une domination blanche sans partage en renforçant et en légitimant l'exploitation de la main d'œuvre servile. Une idéologie raciale paternaliste justifiait des interactions asymétriques caractérisées par un mélange de grande proximité physique et de distance sociale insurmontable entre les races. La seconde phase combinait les conflits de classe entre les Blancs et l'oppression raciale durant la période d'industrialisation du pays (entre 1880 et 1945). Les gigantesques bouleversements économiques et urbains qui accompagnèrent le développement rapide $d u$ capitalisme industriel minèrent l'hégémonie de la classe dominante blanche et entrainèrent des conflits industriels et une concurrence et des tensions accrues entre travailleurs blancs et noirs. Les efforts déployés par les travailleurs blancs pour éliminer la concurrence représentée par la main d'œuvre noire engendrèrent un système élaboré de mesures ségrégatives (le système "Jim (row») renforcé par une virulente idéologie de racisme biologique. L'institutionnalisation de ces nouvelles et plus sévères restrictions raciales réussit en grande partie parce que les machines politiques des grandes villes étaient sous le contrôle des groupes ethniques blancs. Une fois encore, les changements dans le système de production engendrèrent un changement dans les relations raciales alors que le pouvoir d'État joua un rôle décisif dans la consolidation de la domination blanche. Selon Wilson ${ }^{6}$, au cours de la période d'après-guerre se produisit «une transition progressive des inégalités raciales vers les inégalités de classe » qui se cristallisa complètement après les années soixante. La caractéristique distincte de cette période est une bifurcation spectaculaire du sort des Noirs de la classe moyenne et de la classe ouvrière : alors que les premiers profitèrent de la demande accrue d'employés salariés, de la pression gouvernementale en faveur de la discrimination positive, et de la croissance de l'emploi privé et public, les seconds se trouvèrent bloqués aux échelons inférieurs de l'emploi industriel alors que les emplois manuels sans qualification étaient détruits en masse par les innovations technologiques, les délocalisations industrielles et le déplacement sectoriel vers les services. Mais le changement le plus spectaculaire de cette période a sans doute été la «déracialisation » des institutions politiques : alors que dans le passé l'État était « clairement un instrument au service de 
la population blanche pour l'oppression des noirs", il s'attachait désormais "à promouvoir l'égalité raciale. $»^{7}$.

7 Deux thèses subsidiaires découlent de cette argumentation historico-structurelle. Premièrement, le lieu où s'exprime le conflit racial s'est déplacé du domaine économique au domaine sociopolitique. Selon Wilson ${ }^{8}$, les effets combinés de la décentralisation et de la restructuration industrielles et des politiques publiques racialement progressistes ont pratiquement éliminé les conflits raciaux dans le domaine économique. Ces conflits se centrent désormais sur les questions du contrôle de l'espace, des écoles de quartier et des appareils politiques locaux plutôt que sur l'accès à l'emploi. Deuxièmement, du fait des divisions de classes croissantes qui sont récemment apparues au sein de la communauté noire, parler d'une expérience noire uniforme n'a guère de sens. En particulier, la capacité des Noirs de la classe moyenne - ceux qui occupent des emplois qualifiés dans les services ou exercent des professions intellectuelles supérieures - de profiter des nouvelles opportunités professionnelles créées par les programmes de discrimination positive, et de protéger leurs enfants de la mobilité sociale vers le bas, forme un contraste saisissant avec l'incapacité fondamentale des pauvres des ghettos à simplement accéder au travail salarié et à s'assurer les moyens de sortir et de sortir leurs familles de la pauvreté persistante.

8 Il faut souligner que Wilson n'affirme ni ne suggère à aucun moment que les préjugés raciaux appartiennent au passé et que les États-unis sont aujourd'hui la société indifférente à la race que ses idéologues célèbrent. En fait, il prend la peine de préciser que « lorsque je parle de l'importance décroissante de la race, je n'ignore pas l'héritage de la discrimination passée et n'affirme pas non plus que la discrimination raciale n'existe plus $»{ }^{9}$ S'il accorde peu d'attention aux nouvelles formes plus subtiles de discrimination dans la période contemporaine, c'est qu'il pense que les racines de l'inégalité raciale ne résident plus dans des pratiques discriminatoires intentionnelles motivées par la couleur de peau. Elles sont plutôt à rechercher dans des mécanismes économiques impersonnels et indifférents à la race qui polarisent les Noirs et pénalisent prioritairement ceux qui sont au bas de la hiérarchie sociale. Ce sont les changements macro-économiques, et non les préjugés raciaux, qui donnent la clé pour comprendre le sort du sous-prolétariat noir enlisé dans les centres-villes délabrés. Les programmes fondés sur la race visant à promouvoir l'égalité raciale profitent avant tout à la classe moyenne afro-américaine et non aux Noirs pauvres dont la situation se détériore rapidement. L'objectif des politiques sociales devrait donc être d'affronter directement « les aspects omniprésents et destructeurs de la subordination de classe » qui vont bien au-delà de la discrimination raciale. ${ }^{10}$

9 Dans ce sens, "l'importance décroissante de la race» est une formule aussi provocatrice que trompeuse, car Wilson n'affirme en aucun cas que la race est devenue insignifiante, ou même moins signifiante en termes absolus. Il soutient simplement que l'inégalité raciale est aujourd'hui le produit des décalages entre différentes structures spatiales, économiques et sociales, et que la position de classe, un facteur mineur dans le passé, a maintenant une importance fondamentale au sein de la communauté noire, comme c'est le cas depuis longtemps parmi les Blancs. En fait, un titre bien plus approprié pour son livre aurait été « l'importance croissante de la classe ». 


\section{La controverse : l'objet ou rapport à l'objet?}

10 J'ai déjà signalé que l'expression « importance décroissante de la race » est un raccourci trompeur de la théorie de Wilson. Pourtant ses commentateurs et critiques s'en sont vite emparés pour le soumettre à un violent feu rhétorique - dégénérant souvent en attaques ad hominem. Ce qui m'a frappé d'emblée lorsque j'ai pris connaissance de cette controverse c'est la façon dont la plupart des critiques de Wilson, et particulièrement les universitaires noirs, ont concentré leur attention sur la partie de sa thèse portant sur la " classe moyenne » et passé pratiquement aux oubliettes son modèle historique ou ses arguments concernant les Noirs pauvres. Ils ont consacré l'essentiel leur énergie à réfuter l'assertion supposée de Wilson selon laquelle les Noirs de la classe moyenne ne souffraient plus de discrimination et avaient "réussi » à l'instar de leurs homologues blancs. Il y a un élément incontestable d'autodéfense dans la vigueur, la fureur même, avec laquelle les chercheurs en sciences sociales afro-américains se sont efforcés de débusquer et de dénoncer la moindre allusion de Wilson aux "privilèges" des Noirs éduqués, tout en ignorant presque entièrement la pièce maîtresse de sa théorie. De te fabula narratur : si cet élément du modèle de Wilson - qui est à mes yeux subsidiaire - a attiré provoqué un tel mécontentement et un tel malaise intellectuels, c'est bien qu'il dérangeait ses critiques, non pas en qualité de chercheurs, mais en tant que personnes, parce qu'il remettait en question la compréhension qu'ils ont d'eux-mêmes. Une analyse même superficielle des positions sociales et universitaires des protagonistes révèle qu'une bonne partie de la controverse autour de «l'importance décroissante de la race » trouve son origine, non dans l'objet lui-même, mais bien le rapport contrarié que différents analystes entretiennent avec l'objet, rapport qui décrit une large gamme allant de la fierté au du ressentiment en passant par l'envie, l'insécurité et la culpabilité, le plus souvent inconscients.

11 L'une des raisons majeures qui font que le débat sur le couple "race et classe » a jusqu'ici engendré une controverse aussi virulente que peu éclairante est l'absence flagrante de réflexivité de la part des participants, soit leur incapacité à faire le départ entre l'objet et le rapport à l'objet de sorte à faire entrer ce rapport dans l'énigme à résoudre. ${ }^{11}$ En tant qu'universitaires noirs, la plupart des protagonistes du débat sont eux-mêmes impliqués personnellement et scientifiquement et, qu'ils le réalisent ou non, ils défendent des intérêts académiques et personnels. Ils s'efforcent, consciemment ou non, de protéger une image d'eux-mêmes en tant qu'universitaires et en tant qu'individus afro-américains. Une bonne part de leur attitude sur cette question résulte donc d'une projection, dans l'objet d'étude, de leur rapport à cet objet, c'est-à-dire de leur expérience particulière du nœud «race et de classe » dans leur trajectoire biographique et académique.

12 Mais il y a une seconde dimension à cette réflexivité. Avec un minimum de recul, il est facile de discerner que les théories concernant la race, la classe ou tout autre identité sociale (citoyen, femme, membre d'une communauté religieuse, etc.) susceptible de fonder la revendication d'une part des ressources collectives sont, à des degrés divers, à la fois des réponses à des perceptions sociopolitiques préexistantes des réalités ethnoraciales et des efforts visant à influencer ces perceptions. ${ }^{12}$ En effet, la violence du débat sur l'état du couple "race et classe » ne provient pas seulement du fait qu'il affecte profondément les participants dans leur propre identité ; il exerce également un effet sur la réalité elle-même. On a souvent souligné que les théories de la race ont une 
dimension fortement normative et s'articulent, délibérément ou non, aux questions de politique publique. ${ }^{13}$ Cela tient à ce que les structures symboliques produites par les universitaires, à savoir les taxonomies de race et de classe qu'ils élaborent sont, comme toutes les structures cognitives, dotées d'un pouvoir constitutif, un world-making power comme l'explique le philosophe Nelson Goodman, ${ }^{14}$ et qu'elles peuvent, sous certaines conditions sociales, aider à façonner la réalité qu'elles prétendent simplement décrire.

Il n'est donc guère surprenant que la cible principale de la controverse autour de The Declining Significance of Race ait été ce que les critiques de Wilson ont interprété à tort comme ses implications "néo-conservatrices" pour les politiques publiques. Par exemple, lors du congrès annuel de 1978 de l'American Sociological Association,l'Association des sociologues noirs s'est empressée de voter une résolution condamnant le livre de Wilson en des termes lourdes d'émotion et d'ignation morale :

Les membres de l'Association des sociologues noirs sont outrés de la représentation erronée de l'expérience noire. Nous sommes également extrêmement perturbés par les implications politiques qui peuvent découler de cet ouvrage et qui, vu la nature de la société étatusnienne, sont susceptibles d'entraîner des décisions tout aussi dommageables en termes de financement, de recherche et de formation. ${ }^{15}$

Mon argument est que tous les discours théoriques sur le couple « race et classe » ont une dimension performative incontournable. Ils remplissent des fonctions à la fois descriptive et prescriptive, cognitive et politique. A travers eux, les chercheurs s'efforcent, de façon plus ou moins consciente, de façonner la réalité en «faisant des choses avec les mots " pour utiliser l'expression pertinente du philosophe John Austin. ${ }^{16}$ En effet, nous avons à faire ici à un ensemble de rapports matériels et symboliques à propos desquels ce que Pierre Bourdieu appelle «l'effet de théorie» est particulièrement puissant. C'est pourquoi la lutte au sein du champ universitaire pour imposer la classe ou la race comme facteur dominant des clivages collectifs dans la société étatsuniennne a été marquée par tant d'acrimonie et d'échanges excessivement amers et passionnés. Sciemment ou non, les chercheurs noirs se voient et voient leurs rivaux comme les porte-parole de leur communauté et ils défendent la position scientifique conforme à ce qu'ils estiment être les intérêts de cette dernière (selon leur position et trajectoire en son sein). Ce qui est en jeu dans cette confrontation, ce n'est donc pas une sorte de "vérité » socialement neutre qui pourrait être découverte de façon indépendante par un contact direct et inaltéré avec les « faits » nus des inégalités raciales et de classe, mais le monopole de l'autorité légitime de dire la vérité à propos de la communauté noire et de sa place changeante au sein de la société étasunienne. Les chercheurs se sont engagés dans cette polémique en partie pour tenter de changer ou de préserver le monde (et leur « sens de leur place» dans ce monde, pour emprunter l'expression de Goffman) en changeant ou en préservant sa représentation (savante). ${ }^{17}$

La controverse déclenchée par The Declining Significance of Race nous offre ainsi une illustration frappante de la lutte symbolique pour imposer une vision scientifiquement légitime de la race et une estimation juste de son poids. La virulence même de ce débat, pour partie académique et pour partie politique, prouve combien les intérêts spécifiques des universitaires interfèrent avec la recherche et la façonnent - ils en sont une partie intégrale. C'est là un élément indéniable d'auto-analyse. Deuxièmement, elle révèle la contribution cruciale du travail symbolique à la construction des groupes, mêmes ceux apparemment évidents comme les communautés raciales dans la société étasunienne. Car si les théories scientifiques du couple « race et de classe » étaient sans effet sur la réalité qu'elles prétendent décrire, si cette dernière était faite d'entités 
naturelles qui évoluent indépendamment de la perception que nous en avons, alors à quoi bon discuter ? C'est précisément à cause du pouvoir de l'« effet de théorie » que cette controverse s'est développée.

Dans la mesure où ce débat à une dimension foncièrement réflexive, une théorie adéquate de la race et de la classe doit elle aussi être réflexive. Elle doit retourner sur elle-même ses outils théoriques et conceptuels afin de montrer qu'elle fait partie de l'objet même qu'elle prétend saisir et souligner comment, en retour, elle contribue à façonner cet objet tout en étant façonnée par lui. Il deviendra alors évident que "l'importance de la race » est avant tout l'enjeu d'une lutte entre universitaires et la forme et le contenu des positions adoptées sur cette question dépendent pour partie des intérêts spécifiques liés à l'appartenance au champ universitaire et la position occupée par les protagonistes dans ce champ..$^{18}$ Nous devons donc analyser cette polémique, non pour satisfaire une impulsion narcissique, mais parce qu'elle fait partie de la réalité complète de l'objet que nous essayons de construire. Une telle analyse réflexive de l'énigme « race et classe » devrait commencer par soulever les questions: Qui parle et à qui ? A partir de quelle position dans le monde universitaire (petite université ou institution d'élite? Professeur titulaire ou vacataire? Discipline établie ou marginale ? etc.) et à l'issue de quelle trajectoire dans l'espace social ? Même si elles semblent peu pertinentes à première vue, ces questions aideraient à neutraliser l'impact du fait que le sujet est dans l'objet et que l'analyste est assis sous son propre microscope et sous celui de ses rivaux.

\section{Critique de la controverse autour de The Declining Significance of Race}

17 Wilson et ses critiques n'ont pas reconnu cette dimension réflexive de la controverse déclenchée par The Declining Significance of Race. Il s'ensuit qu'ils ont également ignoré le fait que la géométrie variable de la race dans les écrits sociologiques a autant à voir avec les désirs des universitaires d'agir sur le monde qu'avec leur volonté de l'expliquer. Mais ce n'est pas tout. Car ce ne sont pas tant les détails particuliers du débat qui doivent être contestés que les termes mêmes dans lesquels il a été présenté et conduit. Car en posant la question «La race : importance croissante ou décroissante? ? nous sommes menés à croire à tort qu'un problème aussi complexe et aux facettes aussi multiples peut être réduit à une alternative nette, mesurée au moyen univoque de «tests » empiriques rigoureux, et résolue grâce à quelques arguments de bon sens débouchant sur une opinion positive ou négative.

La présupposition implicite derrière une telle question est que la race est une propriété uniforme invariante dont l' « importance » est unidimensionnelle et peut être évaluée par une sorte de mesure purement objective, de préférence quantitative, soit en ellemême, soit en relation à un autre critère social de classement, implicitement la classe. Selon moi, si nous voulons que cette controverse soit progressive et non régressive (au sens de Lakatos), nous devons admettre les trois propositions suivantes :

- La race n'est pas un ens realissimum, une essence immuable qui imprègne nécessairement et de façon uniforme toute la société. C'est un paralogisme que croire que si la race est un facteur déterminant dont l'influence s'accroît ou décline dans un secteur institutionnel alors elle doit nécessairement être tout aussi déterminante, croissante ou décroissante dans la plupart des autres secteurs. Les significations et indicateurs raciaux opèrent de façon 
différente et différentielle selon les divisions de genre et de classe. L' « importance » relative de chacun de ces principes de domination et leur interaction varient également selon les institutions et les régions au sein d'un espace social multidimensionnel.

- La race ne peut être réduite à un facteur objectif qui détermine mécaniquement « à l'insu des agents » leur conduite et leurs chances de vie. La plupart des chercheurs s'inquiètent de constater que, lorsqu'on l'introduit dans une régression statistique, la race semble encore " expliquer » une grande part de l'écart observé entre Noirs et Blancs en matière de revenu, d'éducation, d'accès à la propriété, de taux d'emploi, etc.. - ou, plus précisément, qu'aucune autre « variable de contrôle » n'élimine le différentiel entre les groupes raciaux, qui est donc attribué de façon résiduel à la race, à savoir à la discrimination. Mais la race n'est pas une variable abstraite qui pousse les gens mécaniquement comme la gravité attire les objets vers la sol : la division raciale réside dans un réseau de significations politiquement négociables, une structure symbolique de pouvoir qui doit être activée afin d'être efficace. ${ }^{19}$ Elle est inscrite non seulement dans la distribution inégale des ressources et des pouvoirs matériels, mais aussi dans l'esprit des gens sous forme de taxinomies pratiques et de catégories mentales qui guident leur perception et leur évaluation du monde social et déterminent par ce biais leur action dans ce monde. Ainsi, les expériences subjectives des agents sont loin d'être secondaires dans ce débat. L' « importance » de la race et ses variations ont deux dimensions étroitement liées : une dimension subjective et une dimension objective, qui ne varient pas nécessairement dans le même sens et avec la même amplitude.

- Nous devons abandonner le désir de réduire un espace social multidimensionnel à l'une ou l'autre de ses dimensions, race ou classe.$^{20}$ La croyance persistante dans le fait qu'un seul principe de vision et de division doit nécessairement prévaloir dans le modelage de la réalité sociale, et ainisi « surdéterminer » l'impact des autres bases de catégorisation, mutile la réalité. Contre cette vision, nous devons affirmer que classe et race peuvent être toutes deux agir de concert, et que leur importance augmenter simultanément d'une façon qui tour à tour aggrave ou limite leur influence réciproque, et que les effets produits par la race peuvent varier selon la classe et le genre, tout comme l'impact propre du genre et de la classe est influencé par la division raciale.

Ici, la notion de complexité intervient comme un premier antidote à l'essentialisme. La complexité doit être introduite à trois niveaux: dans notre conceptualisation de la société, dans notre compréhension de la race et de la classe, et dans notre étude de leurs rapports dynamiques. Nous devons reconnaître que la société n'est pas agencée d'un seul tenant et n'est pas non plus structurée autour d'un axe surdéterminant. Nous devons au contraire la concevoir comme une série de champs ou de jeux relativement autonomes, qui se chevauchent pour partie et sont hiérarchiquement connectés, chacun ayant ses propres règles de fonctionnement, ses propres effets et ses hiérarchies spécifiques. ${ }^{21}$ La race peut être décisive dans tel champ et sans effet dans tel autre ; son importance peut diminuer dans l'un et augmenter dans l'autre. Je ne pense pas qu'une procédure acceptable puisse être conçue pour «résumer» ces « importances » diverses selon les régions et secteurs de l'espace social, dans la mesure où les critères pour une telle évaluation sont eux-mêmes l'enjeu et le résultat de luttes autour de la race. Force est donc de reconnaître aussi que la notion de racisme n'épuise pas la discussion sur la division raciale et son poids. ${ }^{22}$ Le racisme peut s'atténuer et sa forme changer sans que la race ne perde nécessairement de son importance. Je pense que c'est actuellement le cas dans la société étasunienne: toutes les mesures des relations actuelles entre Blancs et Noirs indiquent qu'elles sont moins distantes, plus directes et ostensiblement non raciales que dans le passé..... et pourtant la race 
demeure indéniablement, selon l'expression de C. Hughes ${ }^{23}$ " un trait essentiel de statut » définissant les identités et les chances de vie individuelles et collectives aux États-unis. Enfin, notre conception des rapports entre race et classe doit être complexifiée. Ce domaine de recherche a connu une pandémie d'empirisme déplacé, en violation patente de l'injonction d'Aristote appelant à ne pas traiter un sujet avec un degré d'exactitude qu'il ne saurait admettre - autre exemple de ce que Stanley Lieberson appelle « essayer de réaliser l'irréalisable » en sciences sociales ${ }^{24}$. Autrement dit, alors que nos enquêtes empiriques sur le role du facteur racial sont excessivement complexes, en s'appuyant sur des méthodologies ou des modèles d'inférence dont la validité est souvent ténue sinon douteuse, la réflexion théorique sur le couple « race et classe » est demeurée étonnement simple, voire simpliste.

Le plus souvent, la race est traitée comme une simple variable démographique à introduire dans les regressions statistiques, comme si elle été dotée d'une réalité autonome, indépendante de l'action et des expériences des agents sociaux concrets. Contre cette " métaphysique de la structure sociale ${ }^{25}$, nous devons affirmer avec force que l'appartenance raciale est "plus une attitude politico-culturelle qu'une catégorie nette aux frontières clairement définies $»{ }^{26}$ Les participants à ce débat ont rarement exploré la façon dont la race opère dans la réalité, comment les frontières ethnoraciales sont créées et signalées, maintenues ou contestées selon des modèles observables d'action individuelle et collective, ou comment l'identité raciale comme d'autres formes identitaires contribuent à ce processus. Ainsi, Wilson et ses critiques commettent à certain degré les trois erreurs mentionnées ci-dessus. Mais, à mes yeux, c'est l'absence de la notion d'agence et de lutte historiques qui constitue la carence la plus conséquente de l'analyse de l'inégalité raciale contemporaine produite par Wilson, notamment dans The Truly Disadvantaged ${ }^{27}$, le livre qui fait suite et répond aux questions soulevées par The Declining Significance of Race. Les catégories de race, de classe et d'économie ys sont toutes réifiées et transformées en entités opérant de façon autotélique selon une logique qui semble se développer inexorablement à la manière d'un processus naturel. ${ }^{28}$ Les origines politiques et la persistance des inégalités raciales contemporaines (dans les registres du logement, de l'éducation, des programmes sociaux et des politiques urbaines par exemple) sont largement masquées. Et, dans la mesure où les structures économiques sont conçues comme des forces quasiment naturelles, comme « des entités extra-humaines possédant leurs propres lois internes et en cela tout à fait indépendantes de l'action ou de l'inaction humaine ", ${ }^{29}$ la culture doit aussi être réduite au statut d'un simple réflexe de "la structure d'opportunités». Bref, toute notion d'action historique disparait, et les Noirs pauvres sont présentés comme des victimes passives qui se contentent de ré-agir à hue et à dia à des facteurs objectifs sur lesquels ils n'ont aucune emprise et dont personne ne semble être politiquement responsable - c'est ce que l'on pourrait appeler le paralogisme $d u$ mécanisme. Le fait qu'il négligence les luttes et les rapports de pouvoir concrets, au niveau de l'entreprise, de la ville ou de l'État (local ou fédéral) contraint Wilson à formuler son analyse en termes quasi évolutionnistes et fonctionnalistes, selon lesquelles chaque étape de la transformation du système de production engendre l'idéologie et les politiques publiques aptes à le stabiliser. Mais, en réalité, pour prendre l'exemple du système politique, l'État américain a été pour partie dé-racialisé durant les années 1960 et 1970, parce que la mobilisation des Noirs a provoqué une série de changements dans les rapports de force politiques ${ }^{30}$ loin qu'une nécessité fonctionnelle aie soudain dicté de traiter tout le monde à égalité comme des citoyens à part entière. 
Et il est clair que l'État a été de nouveau racialisé avec la montée du reaganisme sur la scène politique. ${ }^{31}$

21 Dans leur réaction à ce qu'ils perçoivent comme une indifférence au racisme, les critiques de Wilson sont, à l'opposé, tombé dans le paralogisme de l'intentionalisme en réintroduisant la notion d'agence dans la théorie raciale sous la seule forme limitée de la discrimination raciale volontaire ou de pratiques motivées par la race dans les rapports entre Blancs et Noirs. Ce faisant, ils négligent le fait qu'il existe une gamme d'actions fondées sur la race, ou liées à la division raciale, autre que la discrimination intentionnelle et que les rapports au sein d'une même population racisée sont tout aussi importants à cet égard que les rapports entre groupes raciaux. Ici une distinction de type wébérien, entre une action déterminée par la race, conditionnée par la race ou se rapportant à la race serait utile. Car il n'est pas besoin qu'une action soit racialement motivée pour qu'elle ait des conséquences différenciées selon les groupes raciaux. Sur ce point, je suis d'accord avec Wilson que la majeure partie des disparités raciales aujourd'hui ne sont pas engendrées par des actions motivées par la race du type de celles constamment mises en avant par ses critiques.

\section{Une approche alternative : construire la classe et construire la race}

22 Je propose que nous allions au-delà de l'essentialisme et des modèles à une seule variable (race ou classe) ou interactionnistes (race et classe) ${ }^{32}$ pour prendre en compte les processus mutuellement imbriqués de structuration et de contestation de la race et de la classe. Pour ce faire, nous pouvons nous appuyer sur les récents développement de la théorie des classes qui ont déplacé leur attention des questions abstraites de structure et de taxonomie pour s'intéresser aux processus matériels et symboliques concrets par lesquelles les classes se construisent. ${ }^{33}$ Ce nouveau courant dans l'analyse des classes met l'accent sur la notion d'agence et de processus de fabrication; il se détourne des questions de composition et de détermination objectives des classes « sur le papier " pour s'intéresser aux luttes à travers lesquelles les classes se constituent comme collectifs efficaces dans la réalité historique. Cette approche suggère une voie féconde pouir re-conceptualiser la relation du couple race et classe. La plupart des participants à la controverse sur «l'importance de la race " continuent de travailler avec des concepts de classe et de race excessivement simples et statiques. Ils traitent les groupes sociaux et raciaux comme des catégories évidentes composées de populations distinctes -comme autant d'entités toutes faites, déjà constituées en tant que telles dans le réel. Contre cette vision fixiste, il faut admettre que tous les groupes, qu'il s'agisse de classes ou de communautés ethnoraciales, doivent être fabriquées, activement construites par un travail symbolique et organisationnel spécifique de regroupement (class-making et race-making). Dans Économie et Société, Max Weber écrit à ce propos:

Il va de soit quue la race ne crée un "groupe" que lorsqu'elle est perçue subjectivement comme un trait commun: cela ne se produit que lorsque le voisinage ou la simple proximité physique de personnes racialement différentes est la base d'une action conjointe (la plupart du temps politique), ou à l'inverse, lorsque des expériences communes des membres d'un même groupe racial sont liées à quelque antagonisme contre les membres d'un groupe manifestement différent. ${ }^{34}$ 
travaux récents de Pierre Bourdieu, Luc Boltanski, Frank Parkin, Jürgen Kocka Sylvain Maresca et Adam Przeworski démontrent qu'il en va de même pour les classes. Race et classe n'existent que si des gens agissent sur la base de ces catégories. Ce sont des modalités concurrentes d'action, des principes de vision et de division, ancrés dans des structures matérielles, à partir desquels les agents sociaux peuvent être identifiés, les individus désignés, leurs subjectivités constituées et des collectifs mobilisés ou démobilisés. En outre, la classe comme la race mène une double existence : chacune a d'abord une existence matérielle, comme système de différences objectives qui peuvent être observées, mesurées sous la forme de distributions de ressources et de biens efficaces; et une existence subjective, en tant que schèmes de perception, d'appréciation et d'action, sous la forme des distinctions symboliques produites et reproduites par le truchement de dispositions socialement déterminées. ${ }^{35}$

Le fait que classe ou race prévale dans la structuration de l'identité et de la réalité sociales dépend de luttes historiquement contingentes menées simultanément dans l'espace théorique (par des intellectuels, universitaires, politiciens et autres spécialistes dans la représentation du monde social comme les journalistes), et dans l'espace social, par des collectifs réels qui sont mobilisés et constitués politiquement autour de questions économiques ou raciales. Laquelle de ces catégories prévaut, où et de quelle façon, dépend de ces affrontements : il y a une lutte pour la race avant qu'il y ait une lutte entre les races. ${ }^{36}$ De même, l'intersection entre race et classe est déterminée d'un côté par la structure objective de l'espace social et, de l'autre, par les stratégies mises en œuvre par les individus et les groupes dans leur effort pour imposer l'une ou l'autre facette de leur identité. En d'autres termes, les races et les classes sont continuellement organisées, désorganisées et réorganisées en tant qu'effets de luttes - économiques, politiques et idéologiques - qui ne sont pas totalement déterminées au niveau de la structure. Bref, nous devons appréhender la race et la classe, et le couple qu'ils peuvent éventuellement former, comme les résultats variables et changeants de l'action collective dans l'histoire. Ni la race ni la classe ne sont des catégories essentielles qui déterminent exclusivement et mécaniquement les processus sociaux. Tous deux sont des systèmes socialement construits de rapports matériels et symboliques. Cela qui ne signifie nullement qu'elles peuvent être construites n'importe comment dans l'esprit des agents ou dans les micro-échanges des relations interpersonnelles. Au contraire : le travail symbolique indispensable pour établir la race ou la classe comme principe subjectif saillant de vision et de division ne peut réussir que s'il correspond aux différences matérielles inscrites dans la réalité objective.

Pour résoudre l'énigme du couple « race et classe » en Amérique, nous avons plus à attendre d'une reconceptualisation de la fabrication de ces principes de vision et de division que d'évaluations statistiques plus sophistiquées de la contribution apportée par la race à l' " explication » des fluctuations de telle ou telle variable dépendante. De tels tests empiriques ne résoudront jamais la question (croire que les sciences sociales progressent en "réfutant " des hypothèses est une illusion positiviste). ${ }^{37} \mathrm{Ce}$ sont les variables indépendantes de la race et de la classe elles-mêmes qui doivent être explorées et élaborées dans leur double réalité, en tant que systèmes de différences objectives enracinées dans la réalité matérielle (ou dans les institutions) et en tant que dispositions et catégories subjectives de compréhension de la réalité (inscrites dans les corps). 


\section{Coda}

L'argument central de cet article est que le problème de « l'importance croissante ou décroissante de la race " est un faux problème qui, ainsi formulé, n'admet pas de réponse scientifique - ou encore une controverse qui est devenue scientifiquement dégénérative plutôt que progressive. Cela parce que la race, en tant que principe de vision et de division du monde social (ou fondement identitaire et déterminant des chances de vie) n'est pas une catégorie unidimensionnelle purement objective qui opère de façon mécanique et indifférenciée dans l'ensemble de la société. En conséquence de quoi, son « importance » ne peut pas être mesurée de façon univoque.

J'ai avancé l'idée que, pour progresser, l'analyse des rapports entre race et classe aux États-unis doit abandonner l'essentialisme naturaliste qui sous-tend la quête du " facteur déterminant unique " (la race ou la classe, "croissant » ou " décroissant »), quête qui révèle plus sur le rapport du chercheur à son objet (à savoir ses expériences personnelles raciales et de classe ainsi que sa position dans le microcosme universitaire) que sur l'objet lui-même. L'ambition épistémique de mesurer, une fois pour toute, l' « importance de la race " est vouée à l'échec dans la mesure où elle est fondée sur une conception naïve du statut ontologique des groupes : ni les races ni les classes n'existent toutes faites dans la réalité. Elles doivent être construites à travers des luttes matérielles et symboliques menées simultanément pour imposer la race ou la classe comme critère dominant de constuction du monde social, et entre les races et les classes (pour autant qu'elles accèdent à la réalité historique).

L'importance de la race dans les rapports sociaux et la conscience collective est le résultat, historiquement contingent et variable selon les secteurs, d'incessantes luttes de classification qui se livrent à la fois dans le champ universitaire et dans l'ensemble de la société et des institutions politiques, au cours desquels les protagonistes s'efforcent d'imposer le critère de construction du monde social qui correspond le mieux à leurs intérêts spécifiques. Ce sont ces luttes elles-mêmes, et non leur résultat à un moment historique donné, qui doivent faire l'objet de nos enquêtes. L'objectif d'une théorie adéquate des rapports entre race et classe n'est donc pas de déterminer ex cathedra lequel de ces deux facteurs l'emporte finalement au risque de "naturaliser " un rapport de force donné, mais de construire un modèle des luttes à travers lesquelles les professionnels de la représentation du monde social (chercheurs, politiciens, avocats, journalistes, etc.) et les mouvements sociaux, les agents collectifs et individuels (raciaux ou non raciaux) réussissent ou non à imposer l'un ou l'autre des critères comme principe légitime prédominant d'identité et d'action.

L'étude de l'interaction entre race et classe doit donc passer de la description de leur impact différentiel à l'explication de leur construction changeante et à l'analyse des rapports de force politiques qui sous-tendent leur « importance ». Elle doit aussi passer de l'anatomie descriptive des formes changeantes de la domination raciale et de classe à l'étude de leur genèse et de leur reproduction par et dans des luttes sociales structurellement déterminées. Ceci qui suppose de déplacer le cœur de l'analyse de la composition à la formation des groupes raciaux et/ou de classe, à savoir de la démographie à la généalogie des collectifs sociaux. 


\section{NOTES}

1. Réponse à Franz Oppenheimer, Seconde assemblée de l'association sociologique allemande, 1912, Max Weber, Gesammelte Aufsätze zur Soziologie und Sozialpolitik (Tübingen : Mohr, 1924), 489. La préparation de cet article a été rendue possible par une bourse Tocqueville de la Fondation Franco-Américaine de Paris.

2. Reynolds Farley, Blacks and Whites: Narrowing the Gap? (Cambridge: Harvard University Press, 1984), 193. La prudence affichée par l'auteur dans sa conclusion est révélatrice : "J'ai examiné de nombreux indices différents concernant le statut des Noirs. J'ai souligné que les changements devaient être interprétés avec prudence. Parfois les données nécessaires ne sont pas disponibles ; parfois les données sont adéquates mais les interprétations ambiguës. Les différences entre les mesures relative et absolue du changement soulèvent des questions qui ne trouvent pas de réponses claires. L'analyse de tous ces indicateurs n'a pas permis de conclure que l'une des trois opinions est correcte et les deux autres fausses. "

3. Cf. Bart Landry, The New Black Middle Class (Berkeley : University of California Press, 1987), pour un portrait descriptif de la "nouvelle classe moyenne noire », et Loïc Wacquant et William Julius Wilson, "The Cost of Racial and Class Exclusion in the Inner City ", Annals of the American Academy of Political and Social Science (janvier1989), 8-25, pour une sociographie du sort des Noirs des ghettos de Chicago.

4. Cf. Joseph R. Washington (dir.), The Declining Significance of Race? A Dialogue Among White and Black Social Scientists (Philadelphie : University of Pennsylvania Press, 1979), pour un échantillon de ce débat et William Julius Wilson, "Academic Controversy and Intellectual Growth", in Matilda White Riley (dir.), Sociological Lives, (Newbury Park: Sage 1988), 73-90, pour une réflexion sur l'impact de cette controverse sur sa recherche et son développement intellectuel ultérieur.

5. Plus précisément, Wilson utilise trois variables indépendantes : le système de production et ses exigences fonctionnelles, les politiques et lois de l'État, et la relation entre ces deux éléments. Pris ensemble, ils déterminent les origines et la forme de la domination raciale, tandis que les idéologies raciales et les croyances collectives évoluent en conséquence.

6. William Julius Wilson, The Declining Significance of Race (Chicago: The University of Chicago Press, 1987), 3.

7. William Julius Wilson, The Declining Significance of Race, 17.

8. William Julius Wilson, The Declining Significance of Race, 111.

9. William Julius Wilson, The Declining Significance of Race, 167. « Ma thèse selon laquelle les rapport raciaux aux États-unis sont passées d'une forme d'oppression économique et raciale à une forme de subordination de classe pour les Noirs défavorisés ne signifie pas que les conflits raciaux ont disparus ou ont substantiellement décliné. Au contraire » (ibid.), 23. "Pour la première fois dans l'histoire étatsunienne, les questions de classe entrent vraiment en concurrence avec les questions de race dans la façon dont les Noirs développent et maintiennent une position de groupe » (ibid), 22. On notera que Wilson écrit « entrent en concurrence » et non «remplacent».

10. William Julius Wilson, The Declining Significance of Race, 154.

11. L'importance de cette distinction pour l'épistémologie sociologique est soulignée dans Pierre Bourdieu, "Critique de la raison théorique », Le Sens pratique, Livre I (Paris : Éditions de Minuit, 1980).

12. Voir Loïc Wacquant, "Making Class: The Middle Class(es) in Social Theory and Social Structure " in Rhonda F. Levine, Rick Fantasia, Scott G. McNall (dir.) Bringing Class Back In (Boulder: Westview Press, 1991), pour une discussion détaillée de cette thèse à propos des théories de la classe moyenne. 
13. Cf. Pierre L. Van den Berghe, Race and Racism : A Comparative Perspective (New York : Wiley and Sons, 1967), et Catherine O'Sullivan See et William Julius Wilson, "Race and Ethnicity", in Neil J. Smelser (dir.), Handbook of Sociology (Newbury Park: Sage, 1988), 223-242.

14. Nelson Goodman, Ways of Worldmaking (Indianapolis : Hackett Publishing, 1978).

15. Cité dans Alphonso Pinkney, The Myth of Black Progress (Cambridge: Cambridge University Press, 1984), 15. C'est moi qui souligne.

16. John L. Austin, How to Do Things with Words (New York: Oxford University Press, 1964).

17. Un exemple paradigmatique de cette interférence du biographique et du politique dans la recherche scientifique est le livre d'Alphonso Pinkney (1984) The Myth of Black Progress, dans lequel l'investigation empirique est totalement subordonnée et même froidement sacrifiée au désir de retirer une fois pour toute à ceux qui soulignent les notions de classe et de convergence sociale l'autorité de parler à propos des Noirs.

18. Je fais là une analogie avec la façon dont Bourdieu analyse les " usages du 'peuple' ». Pierre Bourdieu, « Les usages du 'peuple' », in Idem, Choses dites (Paris : Édition de Minuit, 1987), 178-184. 19. Michael Omi et Howard Winant, Racial Formation in the United States (Boston: Routledge and Kegan Paul, 1986).

20. Certains chercheurs s'évertuent à fondre la race dans la classe alors que d'autres tentent de masquer les différences de classe derrière la discrimination raciale ; d'autres encore invoquent la primauté du genre ou réduisent la race à la culture, aux différences de capital humain ou la nationalité. On pourrait avancer que Wilson tend à réduire la race à la classe (ou à la position économique) tandis que ses opposants ramènent tout à la race en raison des positions respectives qu'ils occupent dans la structure du champ discursif (et académique) au sein duquel ils opèrent.

21. L'idée selon laquelle la société est faite de champs (à savoir des espaces ou des systèmes de positions relativement autonomes définis par des rapports objectifs de force et de lutte autour d'enjeux spécifiques) est développée dans Pierre Bourdieu, « Espace social et genèse des classes ", Actes de la Recherche en Sciences Sociales ( $\left.n^{\circ} 52-53,1984\right)$, et Ibid., "Critique de la raison théorique », Le Sens pratique. Une notion comparable, qui fait de la société un ensemble de jeux se chevauchant, est proposée par Samuel Bowles et Herbert Gintis, Capitalism and Democracy: Property, Community, and the Contradictions of Modern Social Thought (New York: Basic Books, 1986).

22. Le racisme est ici définit comme une idéologie et une pratique d'exploitation et de domination basées sur la couleur de la peau. Cf. William Julius Wilson, Power, Racism and Privilege (New York: The Free Press, 1973).

23. Everett C. Hughes, "Dilemmas and Contradictions of Status ", in Lewis A. Coser (dir.), The Pleasures of Sociology (New York: Mentor [1945] 1980).

24. Stanley Lieberson, Making It Count: The Improvement of Social Theory and Social Research (Berkeley: University of California Press, 1985).

25. Norbert Elias, What is Sociology? (New York: Columbia University Press, 1978), 16.

26. Troy Duster et Barry Wellman, «Le fantôme de Frantz Fanon ", Actes de la recherche en sciences sociales (71/72 : Mars 1987), 136.

27. William Julius Wilson, The Truly Disadvantaged: The Inner City, the Underclass, and Public Policy (Chicago: The University of Chicago Press, 1987).

28. L'absence de la politique et des luttes est une nette régression par rapport à ses travaux antérieurs -notamment William Julius Wilson, Power, Racism and Privilege-- dans lesquels les rapports de pouvoir sont un élément central d'explication des relations raciales. Une sociologie élémentaire de la connaissance suggère que la tendance à minorer le rôle de la politique s'explique pour partie par le changement du public principal de Wilson, de la communauté universitaire aux cercles de décideurs politiques, et par la censure spécifique que le champ discursif de la recherche en politique publique impose à tous ses participants.

29. Norbert Elias, What is Sociology ?, 18. 
30. Frances Fox Piven et Richard A. Cloward, Poor People's Movements: How They Succeed, Why They Fail, (New York: Pantheon Books, 1976); Doug McAdam, Political Process and the Development of Black Insurgency, 1930-1970 (Chicago: The University of Chicago Press, 1982).

31. Michael Omi et Howard Winant, Racial Formation in the United States.

32. Thomas Pettigrew, "New Black-White Patterns: How Best to Conceptualize Them?" Annual Review of Sociology (11: 1985), 329-346.

33. Loïc Wacquant, "Making Class: The Middle Class(es) in Social Theory and Social Structure".

34. Max Weber, Economy and Society (Berkeley: University of California Press, 1978 \{1922\}), vol. 1, 387.

35. Pierre Bourdieu, "Espace social et genèse des classes ", Actes de la Recherche en Sciences Sociales ( $\left.{ }^{\circ} 52-53,1984\right)$.

36. Avec mes excuses pour cet emprunt à Adam Przeworski, Capitalism and Social Democracy (Cambridge et Paris: Cambridge University Press et Editions de la Maison des Sciences de l'Homme, 1985), qui avance cet argument à propos de la classe ouvrière.

37. Par exemple, la même procédure (qui consiste à mesurer l'inégalité résiduelle entre les races après avec contrôlé statistiquement les effets d'une série de variables) fournit des estimations opposées selon que l'on substitue la moyenne blanche dans l'équation concernant les Noirs ou la moyenne noire dans l'équation concernant les Blancs. Sans parler des problèmes de sélectivité (la discrimination indirecte opérant à travers des variables de contrôle), la contagion et la causalité asymétrique. Cf. Stanley Lieberson, Making It Count: The Improvement of Social Theory and Social Research (Berkeley: University of California Press, 1985.

\section{AUTEUR}

\section{LOÏC WACQUANT}

Loïc Wacquant est professeur à l'Université de Californie, Berkeley, et chercheur au Centre de sociologie européenne, Paris. Ses travaux portent sur la marginalité urbaine, l'incarnation, l'Etat pénal et la théorie sociologique. Il est notamment l'auteur de Pierre Bourdieu and Democratic Politics: The Mystery of Ministry (2005), Parias urbains. Ghetto, banlieues, Etat (2006), et Punishing the Poor: The New Government of Social Insecurity (2009). Il est co-fondateur et directeur de la revue transdisciplinaire Ethnography. Pour plus d'informations, voir http://sociology.berkeley.edu/ faculty/wacquant/ 